\section{Antibacterianos naturales orales: Estudios en la Facultad de Odontología de la Universidad Nacional Mayor de San Marcos}

Antibacterial oral natives: Studies in the Faculty of Odontolgy of the National Major University of San Marcos

\section{Resumen}

La caries dental es una enfermedad que afecta al $95 \%$ de la población humana. Uno de los medios para enfrentarla en los tiempos modernos, es el uso de las sustancia naturales, especialmente aquellas que contienes polifenoles, sea: 1) como simple infusión, 2) luego de un tiempo de hervido, y 3) como extracto luego de un proceso químico; que además tienen las ventajas como: fácil acceso y manejo, bajo costo y sobre todo pocos efectos colaterales indeseables. En la Facultad de Odontología de la Universidad Nacional Mayor de San Marcos, se han efectuado varios estudios, tanto in vitro, como in vivo los cuales fueron analizados con el objetivo de proveer un panorama sobre los antibacterianos naturales orales estudiados y continuar los estudios para su posible aplicación preventivas tendientes contribuir a la solución del problema: caries dental. A partir del análisis de los resultados de los estudios se puede citar las siguientes conclusiones: 1) En estudios in vitro, hay evidencias del efecto antibacteriano de los principios naturales (Extracto de Propóleo, Erythroxylum novogranatense, Minthostachys mollis, Camellia sinensis y Croton lachleri i para la flora bucal. Asi mismo, no se observó efecto antibacteriano con: Aloe vera y Uncaria tomentosa. 2) En estudio in vitro, Camellia sinensis ha demostrado capacidad de evitar la formación de placa bacteriana y 3) En estudio in vivo, Camellia sinensis en colutorio mostró efecto antibacteriano oral hasta 30 minutos después del enjuague bucal.

\begin{abstract}
Dental caries is a disease that affects $95 \%$ of the human population. One of the mechanisms to prevent it in modern times, is the use of natural substances, specially those that contain polyphenols: 1) like a simple infusion, 2) after a boiled time, and 3) like an extract after a chemical process. They also have advantages such as easy access and management, low cost and, most of all, few undesirable side effects. In the Faculty of Odontology of the National Major University of San Marcos, several studies have been carried out in vitro and in vivo. They were analyzed to search for evidence in order to show actual tendencies to possible application for a final solution: dental caries. Final conclusions are: 1 ) In vitro studies, there are evidences of the anti-bacterial effect of the natural principles (Propóleo, novogranatense Erythroxylum, Minthostachys mollis, Camellia sinensis and Croton lachleri extracts) for the oral flora. Also, anti-bacterial effect was not observed with: Aloe vera and Uncaria tormentosa. 2) In vitro studies, Camellia sinensis has demonstrated capacity to avoid bacterial plaque formation. 3) In vivo study, Camellia sinensis in colutorio showed oral anti-bacterial effect up to 30 minutes after mouthwash.
\end{abstract}

ODONTOLOGIA SANMARQUINA

ISSN: $1560-9111$

ARTículo de ReVISIÓN

\section{Hilda Moromi, ${ }^{1}$ Elba Martinez Cadillo, ${ }^{2}$ Donald Ramos Perfecto. ${ }^{2}$}
Mg. Profesor Principal del Dpto. de Ciencias Básicas. Jefe del Laboratorio de Microbiologia.
Docentes del Dpto de Ciencias Básicas. Laboratorio de Microbiología.

\section{Correpondencia:}

Hilda Moromi Nakata

La Verbena 377. La Molina

Teléfono: 996175270

Correo electrónico: hmnbio@hotmail.com

Palabras clave: Caries dental, antibacterianos naturales orales, estudios in vitro e in vivo, propóleo peruano, Erythroxylum novogranatense, Camellin sinensis, Menthostachys mollis, Croton lachleri.

Key words: Dental caries, antibacterial oral natural, in vitro studies and in live, propoleo peruvian, Erythroxylum novogranntense, Camellia sinensis, Menthostachys mollis, Croton lachleri.

\section{Introducción}

En la cavidad oral habitan mas de 500 especies de bacterias, en niveles de $10^{8}$ - $10^{9}$ bacteria por $\mathrm{ml}$ de saliva o $\mathrm{mg}$ de placa dental. ${ }^{1}$

La caries dental es aún el gran problema en la salud bucal en todas la latitudes mundo. En el Perú el Ministerio de salud, notifica una prevalencia de $95 \%$ caries dental. ${ }^{2}$ El biofilm de la placa dental está íntimamente ligado a tal patología, siendo el Streptococcus mutans, el microorganịismo más importante seguido por el Lactobacillus y el Actinomyces. ${ }^{3,4}$ De ahr que el control de los microorganismos relacionados con la plasa dental, así como del proceso carioso, es de vital importancia para el control de la enfermedad, y uno de los mecanismos es el uso de antimicrobianos. ${ }^{4,5}$

La medicina natural, a partir de las plantas y sus propiedades antimicrobianas, últimamente ha recibido mucha atención de los científicos, comprobando una series de propiedades de compuestos como los polifenoles, que van confirmando que permiten combatir a los agentes patógenos como el Stapliylococcus aureus resistentes, ${ }^{6,7} \mathrm{y}$ adicionalmente a otras bacterias buca- les como S. mutans, ${ }^{8,9}$ y Porphyromomas gingivalis, referido por Okamoto; ${ }^{10}$ así como la actividad enzimática proteinica importante que afecta los tejidos periodontales. ${ }^{11}$

Las ventajas son diversas: fácil acceso y manejo, bajo costo y sobre todo pocos efectos colaterales indeseables.

Entre los fito principios que ofrece mayores ventajas, es el té, especialmente el verde y el blanco, por la presencia de taninos que inhibe la síntesis del dextran, ${ }^{12}$ el fluor que inhibe al acción enzimática, así como los flavonoides que inhibe la adherencia, la inhibición 
de la producción de acido láctico ${ }^{13}$ por los extractos, entre otros, confieren efecto inhibitorio en el desarrollo del S. mutans, y también sobre los hongos, conocimientos que han propiciado la adición de los extractos en algunas pastas dentales ${ }^{14}$ a tal punto que la Sociedad Americana de Microbiología, en su encuentro del 2004 con los investigadores de la Universidad de Pace de Nueva York, concluyen que los extractos de té verde matan las bacterias y que por tal característica ya puede se incluido dentro de las alternativas para la higiene oral. Sin embargo están también las otras sustancias naturales, que se refieren y destacan más adelante.

El objetivo de la publicación es proveer un panorama cuantitativo $y$ cualitativo de la ciencia y la tecnología sobre los antibacterianos naturales orales; y brindar elementos para el análisis de las tendencias en este campo. En tal sentido, comprende un relevamiento de los hallazgos de conocimientos básicos que sobre el tema se han conseguido en la Facultad de Odontología de la Universidad nacional Mayor de San Marcos, y ofrecer informaciones referentes para la ejecucion de investigaciones preventivas tendientes a la solución del problema: caries dental.

\section{Método}

Se realizó la investigación bibliográfica a partir de Tesis de Grado y Artículos publicados en la Revista Odontología sannarquina, desde los cuales, los conocimientos fueron analizados $y$ sintelizados en función a los objetivos planteados para ésta revisión.

Las evidencias analizadas comprenden los estudios in vitro e in vivo, de los principios naturales, en tanto antibacterianos orales, en las modalidades de uso: como efecto de la dilución de los extractos, y como cfecto de la infusión inmediata.

\section{Estudios in vitro;}

El análisis del referente: tamaño del diámetro del halo de inhibición (Fig 1) de los estudios in vitro, muestran que los halos de las sustancia naturales frisan entre los $10-20 \mathrm{~mm}$, y en el caso de Croton lachleri llega a $30 \mathrm{~mm}$ ); comparados con el $15 \mathrm{~mm}$ de la Clorhexidina y el $33-68 \mathrm{~mm}$ de la Amoxicilina; demostrándose la evidente efectividad antibacteriana de las sustancias naturales.
Otra notoria evidencia es la acción de Muña: efectiva bajo la forma de extracto, pero inefectiva en la foma de infusión al $10 \%$.

\section{a. Estudio con extracto de propóleo}

Usando cepas patrones en cultivos ad hoc, adicionadas con tres diluciones

Cuadro 1. Diámetros de los halos de inhibición en $\mathrm{mm}$ in vitro del efecto antibacteriano del extracto etanólico del Propóleo peruano ${ }^{15}$

\begin{tabular}{lccccccccccc}
\hline & \multicolumn{1}{c}{ Dilución porcentual del Extracto Propoleo } & \multirow{2}{*}{ Clorhexidina } & \multicolumn{2}{c}{ Testigo } \\
\cline { 2 - 11 } Bacteria & \multicolumn{2}{c}{0,8} & \multicolumn{2}{c}{20} & \multicolumn{2}{c}{30} & & & & \\
& \cline { 2 - 10 }$y$ & Prom & $\%$ & Prom & $\%$ & Prom & $\%$ & Prom & $\%$ & Prom & $\%$ \\
\hline S. mutans & 13,56 & 141,1 & 12,00 & 124,9 & 12,33 & 128,3 & 11,72 & 121,0 & 9,61 & 100 \\
L. casei & 11,25 & 126,4 & 10,10 & 113,5 & 11,20 & 125,8 & 10,26 & 115,3 & 8,90 & 100 \\
\hline
\end{tabular}

b. Estudio con el extracto alcohólico de Erythroxylum novogranatense (Coca)

Cuadro 2. Diámetros de los halos de inhibición en $\mathbf{m m}$ in vitro por efecto del extracto alcoholico de Erythroxylum novogranatense sobre la flora bacteriana salival mixta ${ }^{16}$

\begin{tabular}{lcccc}
\hline Concentración & Promedio* & D.S & Mínimo & Máximo \\
\hline $250 \mu \mathrm{g}$ & $10,96^{\star}$ & 1,20 & 9,25 & 12,75 \\
$500 \mu \mathrm{g}$ & $12,9^{\mathrm{b}}$ & 0,99 & 10,50 & 13,75 \\
$1000 \mu \mathrm{g}$ & $13,46^{\mathrm{*}}$ & 0,87 & 11,75 & 14,75 \\
$1500 \mu \mathrm{g}$ & $14,71^{\mathrm{d}}$ & 0,87 & 13,00 & 16,50 \\
\hline
\end{tabular}

*Hay diferencia estadística, cuando las letrillas son diferentes

\section{c. Estudio del extracto de Minthostachys mollis (Muña)}

Cuadro 3. Diámetros de los halos de inhibición en num in vitro por efecto del extracto de Mintilostachys mollis sobre la flora bacteriana ${ }^{17}$

\begin{tabular}{lcccc}
\hline \multirow{2}{*}{ Bacteria } & \multicolumn{2}{c}{ Minthostachys mollis } & \multicolumn{2}{c}{ Amoxicilina } \\
\cline { 2 - 5 } & $\begin{array}{c}\text { Diam. en } \\
\mathrm{mm}\end{array}$ & D.S & $\begin{array}{c}\text { Diam. en } \\
\mathrm{mm}\end{array}$ & D.S \\
\hline Streptococcus mintons & 16,50 & 5,28 & 68,60 & 2,16 \\
Lattobncillus sp & 14,38 & 2,93 & 42,27 & 2,22 \\
F. nucleatum & 20,13 & 1,31 & 50,50 & 6,45 \\
Actinobncillus actinonit- & 18,42 & 3,98 & 51,58 & 3,73 \\
cetenconitnns & 11,00 & 0,41 & 33,25 & 2,63 \\
Actinomyses sp & & & & \\
\hline
\end{tabular}

\section{d. Estudio de la infusión de Camellia sinusis (Tè verde) y Menthostadtys mollis}

Cuadro 4. Diámetros de los halos de inhibición en $\mathrm{mm}$ in vitro por etecto de la infusión de Cantellin sinensis y Menthostachys nollis sobre la flora bacteriana salival mixta ${ }^{18}$

\begin{tabular}{lcccc}
\hline \multicolumn{1}{c}{ Infusión } & Promedio & D.S & Mínimo & Máximo \\
\hline Cantlin sinensis & 15,27 & 1,72 & 11,00 & 18,00 \\
Mentiostachys mollis & 0,00 & 0,00 & 0,00 & 00,00 \\
Canellia sinensis + & 12,70 & 2,07 & 8,00 & 15,00 \\
Menthostachys mollis & 14,85 & 2,57 & 10,00 & 20,00 \\
Clorhexidina & 0,00 & 0,00 & 0,00 & 00,00 \\
Testigo (agua) & & &
\end{tabular}




\section{e. Analisis comparativo de los estudios in vitro}

Cuadro 5. Diámetros de los halos de inhibición en $\mathrm{mm}$ in vitro del efecto antibacteriano oral de sustancias naturales

\begin{tabular}{|c|c|c|c|c|c|c|c|c|c|c|}
\hline \multicolumn{7}{|c|}{ Extracto } & \multicolumn{4}{|c|}{ Infusión $^{19}$} \\
\hline $\begin{array}{c}\text { Propóleo }^{15} \\
(0,8 \mu g)\end{array}$ & \multirow{2}{*}{$\begin{array}{c}\text { Coca }^{16^{*}} \\
(1500 \\
\mu \mathrm{g})\end{array}$} & \multicolumn{5}{|c|}{ Muña $^{17}$} & \multirow{2}{*}{ Té18 } & \multirow{2}{*}{ Té } & \multirow{2}{*}{ Muña } & \multirow{2}{*}{$\begin{array}{l}\text { Té + } \\
\text { Muña }\end{array}$} \\
\hline S. $m^{* *}$ L.c c $^{* *}$ & & S.m $m^{* *}$ & L.sp $\mathrm{p}^{* *}$ & F. $n^{* *}$ & A. $a^{* *}$ & A.sp $p^{\star \star}$ & & & & \\
\hline $13,52 \quad 14,71$ & 14,71 & 16,50 & 14,38 & 20,13 & 14,42 & 11,00 & 10,43 & 15,27 & 0,00 & 12,70 \\
\hline
\end{tabular}

*Flora bacteria salival mixta

${ }^{*}$ S.m $=$ Streptococcus mutans. L.c $=$ Lactobacillus casei. L.sp $=$ Lactobacillus sp. F.n $=$ F. nucleatum. A.a = Actinobacillus actinomecetencomitans. A. $\mathrm{sp}=$ Actinomyces sp.

\section{f. Fito sustancias en pasta dental experimental}

Cuadro 6. Diámetros de los halos de inhibición en $\mathrm{mm}$ in vitro del efecto antibacteriano oral de fito sustancias naturales ${ }^{20}$

\begin{tabular}{lcccccrc}
\hline \multicolumn{1}{c}{ Bacteria } & Sab $^{a}$ & Uña $^{b}$ & SG $^{c}$ & $\begin{array}{c}\text { Sab }+ \\
\text { Uña }\end{array}$ & $\begin{array}{r}\text { Sab } \\
\text { SG }\end{array}$ & $\begin{array}{c}\text { Uña } \\
\text { SG }\end{array}$ & $\begin{array}{c}\text { Sab }+ \\
\text { Uña }+ \\
\text { SG }\end{array}$ \\
\hline Actinomyces $s p$ & 00 & 00 & 16 & 00 & 13 & 12 & 8 \\
Lactobncillus $s p$ & 00 & 15 & 30 & 00 & 28 & 25 & 28 \\
Streptococcus salivarius & 00 & 00 & 10 & 00 & 8 & 8 & 00 \\
Eikenella $s p$ & 00 & 00 & 32 & 00 & 30 & 30 & 32 \\
\hline
\end{tabular}

"Sábila $=$ Aloe vera. "Uña de gato $=$ Uncaria tomentosa. ${ }^{~}$ Sangre de Grado $=$ Croton lachleri.

g. Infusión de Camellia sinensis (té verde) en la formación de la Placa bacteriana por Streptococcus $m u$ tans $^{21}$

Cultivos de $S$. muttans sin adición de Té verde mostraron formación de Placa bacteriana adherida fuertemente en el alambre; en tanto que los cultivos con adición de infusión de té verde mostraron muy poca formación de Placa, y los residuos formados tenían muy poca adherencia, con desprendimiento rápido, Fig 2.

El hallazgos es una evidencia más, de las propiedades del té verde, como antitumoral, antimicrobiano, antimicótico. ${ }^{6}$ Donde los polifenoles del té,

Cuadro 7. Recuento total de bacterias en Agar Tripticasa Soya colectadas en saliva luego de la infusión del Té verde ${ }^{22}$

\begin{tabular}{lrrrrr}
\hline \multirow{2}{*}{ Parâmetro } & \multicolumn{4}{c}{ Unidades formadoras de colonias / $\mathrm{mL}$} \\
\cline { 2 - 6 } & \multirow{2}{*}{ Antes } & \multicolumn{2}{c}{$\begin{array}{c}\text { Inmediatamente } \\
\text { Después }\end{array}$} & \multicolumn{2}{c}{30 minutos después } \\
\cline { 2 - 6 } & & $\mathrm{n}$ & $\%$ & $\mathrm{~N}$ & $\%$ \\
\hline Promedio & 16648062,5 & 12831250,0 & 77,1 & 9743750,0 & 58,9 \\
D.S & 7332722,0 & 7410658,3 & & 7493762,5 & \\
\hline
\end{tabular}

\section{Discusión}

Los efectos antimicrobianos para el control de microorganismos orales mediante sustancias naturales, se estudia desde décadas anteriores ${ }^{23}$ se consideran como inhibidores de la adherencia bacteriana del S. mutans, y el extracto puede inhibir la actividad de los microorganismos cariogénicos por la reducción de la producción de ácidos. $^{7-9}$

\section{Estudios in vivo:}

Estudio con la Camellia sinensis (Té verde)

En el Cuadro 7, el colutorio de la infusión empleado ha mostrado efectividad en la reducción del recuento de las bacterias orales, tanto al registro inmediato a su empleo $(22,9 \%)$ como también al registro luego de los 30 minutos de utilización $(41,1 \%)$. cobrando cada vez una mayor atención, especialmente en lo referente a la acción de los polifenoles (catequinas). En este marco los hallazgos citados, permiten afianzar los conocimientos sobre la materia, evidenciadas por los efectos mostrados tanto in vitro, como in vivo. Por ejemplo la evidencia más notoria es el efecto del extracto de la Muña y no así de la infusión. Es también saltante el efecto de la infusión del Té verde bajo la modalidad de un sólo enjuagatorio, ${ }^{22} \mathrm{y}$ en el deterioro en la formación de la Placa bacteriana. ${ }^{21}$ Estos hallazgos sugieren la necesidad de estudios complementarios, entre los que se incluya, por ejemplo: la frecuencia de enjuagues, otras concentraciones de la infusión, etc.

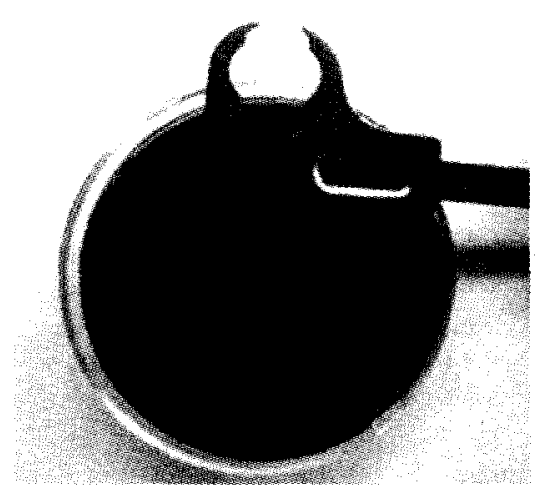

Fig. 1. Halo de inhibición de desarrollo bacteriano.'

Independientemente de la necesidad de mayores estudios, se puede arribar a señalar la aplicación práctica en el uso cotidiano de enjugue bucal con infusión del Té verde,puesto que ahora hay evidencias de otras bondades terapeúticas del Té. Existen diversos mecanismos por los cuales el té verde produce acción sobre las bacterias, entre ellas, la inhibición de la producción de ácidos y de la enzima glucosiltransferasa. ${ }^{24}$

La mayoría de los polifenoles en el té verde son flavonoides, comúnmente conocidos como catequinas. Las principales catequinas son: (-)-epicatechin, (-)-epicatechin-3-gallate, (-)-epigallocatechin, y (-)-epigallocatechin-3gallate. $^{25}$

\section{Conclusiones}

1. En estudios in vitro, hay evidencias del efecto antibacteriano de los principios naturales (Extracto de Propóleo, Erythroxylum novogranatense, Minthostaclys mollis, Cantellia sinensis y Croton lachleri) para la flora bucal. Asi mismo, no se observó efecto antibacteriano con: Aloe vern y Uncaria tomentosa.

2. En estudio in vitro, Camellia sinensis ha demostrado capacidad de evitar la formación de placa bacteriana.

3. En estudio in vivo, Camellia sinensis en colutorio mostró efecto 
antibacteriano oral hasta 30 minutos después del enjuague bucal.

\section{Referencias bibliográficas}

1. Rosan B, Lamont RJ. Dental plaque formation. Microbes Infect. 2000;2:1599. 1607.

2. Ministerio de Salud del Perú: "Plan de Salud Bucal 2005" http://www.minsa. gob.pe/portal/campanas/SBucal/ Archivos/RM538-2005\%20Plan\% 20 de\%20Salud\%20Bucal.pdf

3. www.msnbc.msn.com/id/3079355/ prin/1/displaymode/1098/

4. Negroni M. Microbiología estomatológica. Argentina: Edit. Panamericana. 1999:223-225.

5. Negroni M. Microbiología estomatológica. Argentina: Edit. Panamericana. 1999:237

6. Newson SWB. MRSA: Past, present, future. Journal of Royal Society of Medicine London. 2004;97:509.

7. Dryden MS, Dailly S, Crouch M. A ramdomised, controlled trial of tea tree tropical preparations versus a standard tropical regime for the clearance of MRSA colonization. J. Hosp Infect. 2004:54:283-6.

8. Rasheed A, Haider M. Antibacterial activity of Camellia sinensis extracts against dental caries Arch. Pharm Res 1998 Jun 21 (3):348-52.

9. Otake $S$, Makimura $M$, Kuroki $T$, Nishihara $Y$, Hirasawa M. Anticaries effects of polyphenolic compounds from Japanese green team. Caries Res (Switzerland) 1991;25(6):438-43.
10. Okamoto $M$, Leung $K P$, Ansai $T$, Sugimoto A, Maeda N. Inhibitory effects of green tea catechins on protein tyrosine phosphatase in Prevotello intermedia. Oral Microbiol Inmunol. 2003;18:192-195.

11. Okamoto $M$, Sugimoto $A$, Leung $K P$, Kakayama K, Kamaguchi A, Maeda N. Inhibitory effect of green tea catechins on cysteine proteinases in Porhyromonas gingivalis. Oral Microbiol Inmunol. 2004;19:118-120

12.S. Rosen M, Elvi-Lewis FM, Beck EX. Anticariogenic effects of tea in rats. J. Dent. Res. 1984;63(5):658-660.

13. Koompirojn K, Guay M, Peawchana W, Suesuwan A, Ingkasate. A Inhibition of lactic and poysaccharide formation of Streptococcus mutans by tea extract in Vitro. CU Dent I 2001;24:195-202.

14. Anónimo. Bacteriology; white tea has an inhibitory effect of various pathogenic bacteria. Obesity: Fitness \& Wellness Weeks Atlanta. 2004;Aug 26: 86.

15. Eguizabal AM. Actividad antibacteriana in vitro del extracto etanólico de propóleo peruano contra Streptococcus mutans y Lactobacillus casei. Tesis: Cirujano Dentista. Fac Odontol. Universidad nacional Mayor de San Marcos. 2007.

16. Burrovic RF. Efecto antibacteriano del extracto alcoholico de la hoja de coca de Erythroxylum novogranatense var. Truxillense (Coca) sobre flora mixta salival. Tesis Cirujano Dentista. Fac Odontol. Univ San Marcos. 2006.

17. Díaz LK, Moromi NH. Determinación de la actividad microbiana in vitro de Minthostfichys mollis (Muña) frente a bacterias orales de importancia estomatológica. Odontol Sanmarquina 2005;8(2):3-5

18. Paredes SN. Efecto antibacteriano in vitro de la infusión de Cantellia sinensis y Menthostachys mollis sobre flora salival mixta. Tesis Cirujano Dentista. Fac Odontol. Univ San Marcos. 2006.

19. Moromi NH, Martínez CE, Villavicencio GE, Burga SJ, Ramos PD. Efecto antimicrobiano in Vitro de la Camellin sinensis sobre bacterias orales. Odontol Sanmarquina 2007;10(1):18-20.

20. Gálvez CL, Mendoza RA. Capacidad bactericida de pastas experimentales Anti-A. Odontol Sanmarquina. 2001;1(7):7-16.

21. Moromi NH, Martínez CE. Efecto del tẻ verde en la formación de la Placa bacteriana por Streptococcus mutans. Odontol Sanmarquina. 2006;9(2):23-24.

22. Moromi NH, Martínez CE, Gutierrez IE, Ramos PD, Nuñez LM, Burga SJ, Tello J. Trebejo I. Efecto antimicrobiano in vino de la infusión de Camellia sinensis sobre bacterias orales. Odontol Sanmarquina 2007;10(2):12-14.

23. Saeki $Y$, Ito $Y$, Shibata $M$, Sato $Y$, Okuda K, Takazoe I. Bull Tokio dent. Coll. 1989; 30(3):129-135

24. Tagashira $M$, Uchiyama $K$, Yoshimura T, Shirota M, Uemitsu N. Inhibition by hop bract polyphenols of cellular adherence and water-insoluble glucan synthesis of mutans streptococci. Biosci Biotechnol Biochem 1997;61(2):332-335.

25. Mukhtar H, Ahmad N. Tea polyphenols: prevention of cancer and optimizing health. Am J Clin Nut. 2000;71(6):16981702.

Fecha de recepción: 25 marzo 2009

Fecha de aceptación: 10 julio 2009 